\title{
Hypoglycemic and antioxidant effect of Tai chi exercise training in older adults with metabolic syndrome
}

This article was published in the following Dove Press journal:

Clinical Interventions in Aging

\author{
Víctor Manuel Mendoza- \\ Núñez' \\ Taide Laurita Arista-Ugalde' \\ Juana Rosado-Pérez \\ Mirna Ruiz-Ramos' \\ Edelmiro Santiago-Osorio ${ }^{2}$ \\ 'Research Unit on Gerontology, FES \\ Zaragoza, National Autonomous \\ University of Mexico, Mexico City, \\ Mexico; ${ }^{2}$ Hematopoiesis and Leukemia \\ Laboratory, Research Unit on Cell \\ Differentiation and Cancer, FES \\ Zaragoza, National Autonomous \\ University of Mexico, Mexico City, \\ Mexico
}

Introduction: The antioxidant and anti-inflammatory effects of Tai chi (TC) exercise training in healthy older adults has been demonstrated. However, there are no studies on this effect in older adults with metabolic syndrome (MetS).

Purpose: The aim of this study was to determine the effect of TC exercise on oxidative stress and inflammatory markers in older adults with MetS.

Methods: A quasi-experimental study was carried out with a sample of 110 older sedentary volunteers with clinical diagnoses of MetS: (i) a control group, $n=50$, of individuals who do not participate in physical exercise, of which 37 fulfilled the entire study protocol, and (ii) an experimental group, $\mathrm{n}=60$, of subjects enrolled in a TC exercise training program (eight-form easy), 5 days a week for 6 months, in sessions of $50 \mathrm{~min}$, under the supervision of a qualified instructor, of which 48 fulfilled the entire study protocol. We measured in both groups (pre- and post-intervention) the following cardiovascular parameters: resting heart rate (RHR), diastolic and systolic blood pressure (DBP and SBP), mean arterial pressure (MAP), RHR-SBP product, RHR-MAP product; glycosylated hemoglobin (HbA1c); oxidative stress markers (superoxide dismutase, total antioxidant status, thiobarbituric acid reacting substances, and oxidative stress score); and inflammation markers (TNF- $\alpha$, IL-6, IL-8, and IL-10).

Results: A statistically significant decrease in HbA1c concentration was observed in the TC group compared with the control group $(p<0.05)$. This group also showed a statistically significant increase in TAS and a decrease in the oxidative stress score $(p<0.05)$. We did not observe changes in the cardiovascular parameters (RHR, DBP, SBP, MAP, RHR-SBP product, and RHR-MAP product) in the TC experimental group compared to the control group.

Conclusion: Our findings suggest that the practice of TC exercise has an antioxidative and hypoglycemic effect in the elderly with MetS.

Keywords: Tai chi, oxidative stress, older subjects, metabolic syndrome, HbAlc

\section{Introduction}

Metabolic syndrome (MetS) is a cluster of biochemical and clinical alterations characterized by insulin resistance, hyperinsulinemia, hyperglycemia, dyslipidemia, arterial hypertension, and obesity, in addition to the fact that MetS manifests in prothrombotic and proinflammatory states. In this sense, it has been shown that MetS has an increase in oxidative stress $(\mathrm{OxS}) .{ }^{1}$ The prevalence of MetS in young adults (20-29 years) is less than $10 \%$; however, in individuals $\geq 60$ years of age, the prevalence increases to more than $40 \%{ }^{2,3}$ MetS in older adults constitutes a risk factor for cognitive impairment and fragility linked to OxS and inflammaging. ${ }^{4,5}$ For this reason,
Correspondence: Víctor Manuel Mendoza-Núñez

Facultad de Estudios Superiores Zaragoza, UNAM, Unidad de Investigación en Gerontología, Guelatao No 66, Col. Ejército de Oriente, 09230 México, DF., México

Tel +52555623072I

Fax +52 5557736330

Email mendovic@unam.mx 
different therapeutic alternatives have been proposed, including the indication of metformin, melatonin, healthy diet, and physical exercise. ${ }^{6-8}$ In this sense, physical exercise has been shown to have a significant effect on weight loss and decreased glucose, cholesterol, and triglyceride levels and a decrease in $\mathrm{OxS}$ and proinflammatory markers in subjects with MetS. ${ }^{9}$

Therefore, different modalities of moderate physical exercise have been proposed as adjuvants for the prevention and control of MetS alterations in older adults, considering the decrease of $\mathrm{OxS}$ and chronic inflammation, among which, the practice of Tai chi (TC) is one of the more recommended forms of exercise. ${ }^{10-12}$

TC is a form of traditional Chinese exercise based on modified martial arts; it is classified as a moderate physical activity because its intensity does not exceed $55 \%$ of the maximum oxygen uptake and $60 \%$ of the maximum individual heart rate. ${ }^{13} \mathrm{TC}$ training has been increased due to its beneficial effects on health, especially in the elderly. This exercise is characterized by a series of smooth and continuous movements, with precise execution of position control, deep breathing, and coordination of movements in the legs, arms, torso, and head. The practice of TC includes moving from one position to another at an equilibrium without stopping while also in a state of relaxation and concentration to achieve balance in movements. ${ }^{14}$

Our research group has identified antioxidant and antiinflammatory effects of the practice of TC exercise in healthy older adults. ${ }^{15,16} \mathrm{~A}$ beneficial effect of this physical exercise on $\mathrm{OxS}$ has also been reported in healthy young women and diabetic adults. ${ }^{17,18}$ However, we do not know the effect of $\mathrm{TC}$ on $\mathrm{OxS}$ and proinflammatory markers in older adults with MetS. For this reason, the purpose of the present study was to evaluate the effect of TC exercise on $\mathrm{OxS}$ and inflammatory markers in older adults with MetS.

\section{Materials and methods}

\section{Subjects and design}

The subjects were older, community-dwelling, Mexican mestizos who had been living in Mexico City for 5 years or more, were sedentary in the last 2 years, and with a clinical diagnosis of MetS, defined according to the criteria established in the Third Report of the National Cholesterol Education Program Expert Panel on Detection, Evaluation, and Treatment of High Blood Cholesterol in Adults. ${ }^{20}$

Informative brochures were distributed in the community specifying the objectives of the study and the admission criteria. A quasi-experimental study was performed with a sample of 150 subjects aged 60-74 years. Those who were taking nutritional antioxidant supplements or antiinflammatory drugs $(n=27)$ were excluded from the study, and 13 subjects refused to participate. All subjects agreed to participate in the study after signing a written informed consent form. The ethics committee of the Universidad Nacional Autonoma de Mexico, Zaragoza Campus, approved the research protocol for this study (IN306213-2).

An outline of the study can be seen in Figure 1. The sample was divided into two groups: (i) a control group (CG) with 50 subjects without physical exercise practice, of whom 37 fulfilled the entire study protocol and (ii) an experimental group (EG) with 60 subjects who performed exercise TC (eight-form easy), ${ }^{19} 5$ days a week for 6 months, in sessions of $50 \mathrm{~min}$, under the supervision of a qualified instructor, of whom 48 fulfilled the entire study protocol. The CG included people who were without physical exercise practice during the last 2 years, as well as the 6 months of this study. In this sense, the CG was constituted by people who self-reported that, at the moment, they did not have enough time to practice physical exercise and therefore could not participate in Tai Chi training; however, they expressed interest in participating in the biochemical tests that were carried out at the beginning and after 6 months and considered the possibility of participating in the program later.

All the participants of both the EG and CGs had the following variables measured at the beginning and after 6 months: dietary intake, anthropometric measurements, restoring heart rate and blood pressure, biochemical parameters (glucose, urate, albumin and lipid profile), plasma thiobarbituric acid-reactive substances, total plasma antioxidant status, red blood cell superoxide dismutase, red blood cell glutathione peroxidase, oxidative stress score (OxS-score) and inflammatory cytokines.

\section{Dietary intake}

All the subjects had a caloric intake between 3,000 and $3,500 \mathrm{kcal}$ per day pre- and post-intervention measured by 24-h dietary total recall; nutrient intake (macronutrient and micronutrient) was analyzed using Food Processor ${ }^{\circledR}$ Nutrition Analysis Software. No subject took antioxidant supplements (vitamins or minerals) for at least 6 months prior to initiation of or during the study.

\section{Anthropometric measurements}

The anthropometric measurements of weight, height and waist circumference were obtained following a standardized protocol after recording the clinical history and conducting 


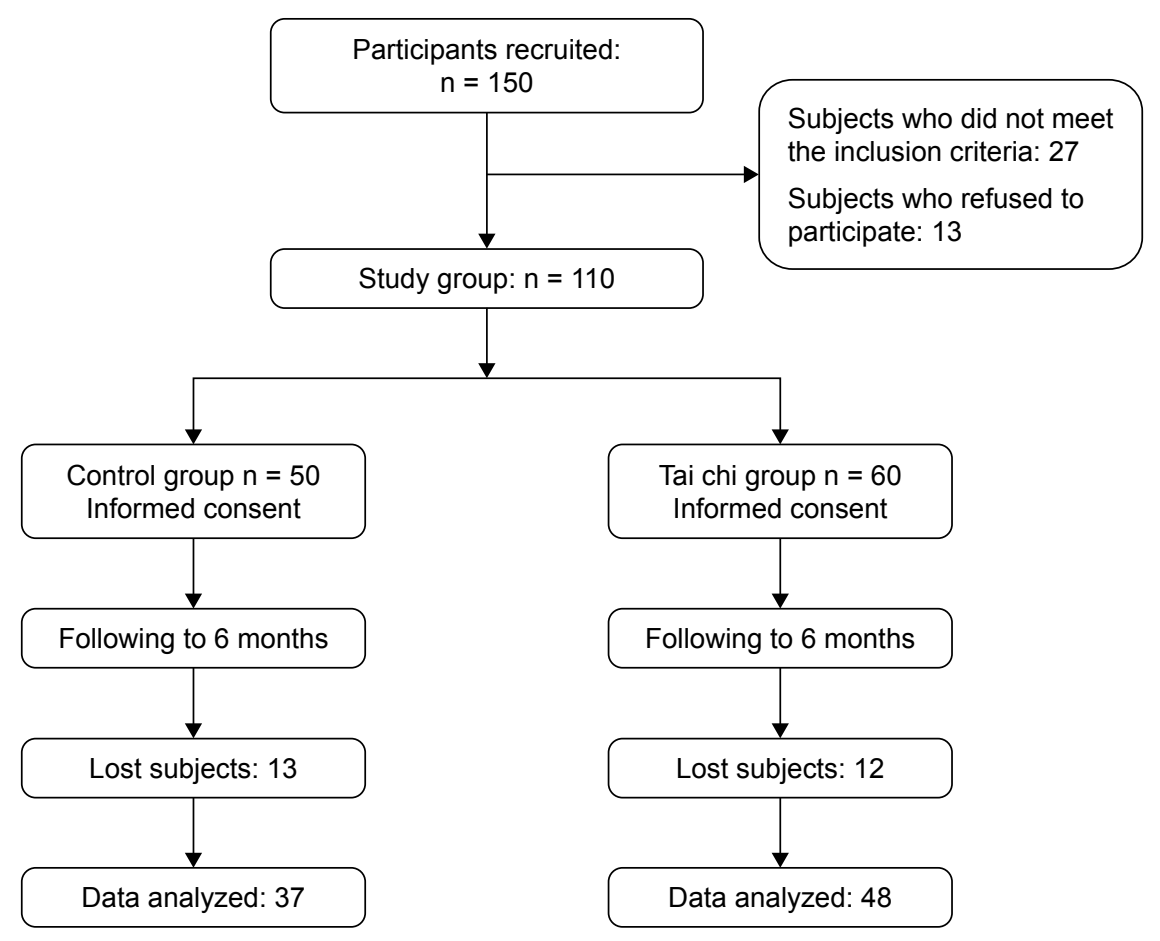

Figure I Outline of the study.

the physical examination. Weight was measured while the subject was wearing underwear and a hospital smock and was in a fasting state (after evacuation). A Torino ${ }^{\circledR}$ scale (Tecno Lógica Mexicana, Mexico City, Mexico), calibrated before each measurement, was used. Height was obtained with an aluminum cursor stadiometer graduated in millimeters. The subject was barefoot with the back and head in contact with the stadiometer in the Frankfurt horizontal plane. The body mass index (BMI) was calculated by dividing weight $(\mathrm{kg})$ by height squared $\left(\mathrm{m}^{2}\right)$. Waist circumference $(\mathrm{cm})$ was measured to the nearest $0.5 \mathrm{~cm}$ with a tape measure at the umbilical scar level.

\section{Resting heart rate and blood pressure}

A 10-second 12-lead electrocardiograph was carried out to measure the resting heart rate (RHR) after the individual had rested in the supine position for $5 \mathrm{~min}$. Blood pressure (BP) was measured following the standardized protocol according to the Official Mexican Norm (Norma Official Mexicana). ${ }^{21}$ Using a mercurial manometer at both arms, BP was registered before the training of TC (BP Baseline) and 6 months after (BP 6 months); measurements were taken in the morning in a fasted condition or $2 \mathrm{~h}$ after breakfast in sitting and standing positions. Subjects with pseudohypertension were identified by applying the Osler technique (feeling the radial pulse when the manometer registered values above the true systolic pressure). Mean arterial pressure (MAP) was calculated as diastolic blood pressure (DBP) plus one third of the difference of systolic blood pressure (SBP) minus the diastolic blood pressure. RHR-SBP product was calculated by $(\mathrm{RHR} \times \mathrm{SBP})$; RHR-MAP product was calculated by $($ RHR $\times$ MAP). Blood pressure was taken by medical technicians who attended training sessions to standardize the procedures. The technicians were supervised to avoid possible biases in measurement.

\section{Blood sampling and biochemical analyses}

Blood samples were collected 1 week before the training of TC and 1 week after 6 months (in both groups, TC and CG) by venipuncture after a 10 -h fasting period and placed in vacutainer/siliconized test tubes without anticoagulant for biochemical determinations (glucose, urate, albumin, lipid profile, and inflammatory cytokines) and with heparin for glycosylated hemoglobin (HbA1c) determination and OxS tests.

Glucose, urate, albumin, cholesterol, triglycerides, and high-density lipoprotein cholesterol (HDL-C) concentration levels were determined using a Merck Vitalab Eclipse autoanalyzer (Merck, Dieren, the Netherlands). In particular, glucose levels were measured by the glucose oxidase method, and urate levels by the uricase colorimetric method. Albumin levels were measured with the bromocresol green technique. 
The low-density lipoprotein cholesterol (LDL-C) was calculated by the Friedewald equation $\mathrm{LDL}=$ total cholesterol - (Triglycerides $/ 5+\mathrm{HDL}) .^{22}$ The reactive $\mathrm{C}$ protein was measured in serum by immunoturbidimetric assay.

HbA1c was measured in a whole blood sample with an immunoturbidimetric assay with an automated Selectra Junior clinical chemistry analyzer.

High and normal control sera were included as quality controls (Randox Laboratories, Ltd). The intra- and interassay variation coefficients were less than $5 \%$ for all determinations.

\section{Plasma thiobarbituric acid-reactive substances}

A thiobarbituric acid reacting substances (TBARS) assay was used as described in Jentzsch et al. ${ }^{23}$ In this assay, one molecule of malondialdehyde reacts with two molecules of thiobarbituric acid to produce a pink pigment with absorption at $535 \mathrm{~nm}$. Amplification of peroxidation during the assay is prevented by the addition of the chain-breaking antioxidant butylated hydroxytoluene. Malondialdehyde standard $(0.2-4 \mu \mathrm{mol} / \mathrm{L})$ was prepared either by hydrolysis of 1,1,3,3-tetramethoxypropane (Sigma-Aldrich Co., St Louis, MO, USA), or the samples (heparinized plasma, $400 \mu \mathrm{L}$ ) were mixed with $400 \mu \mathrm{L}$ of orthophosphoric acid ( $0.2 \mathrm{~mol} / \mathrm{L}$; Sigma) and $50 \mu \mathrm{L}$ of butylated hydroxytoluene ( $2 \mathrm{mmol} / \mathrm{L}$; Sigma-Aldrich Co.); then, $50 \mu \mathrm{L}$ of thiobarbituric acid reagent $(0.11 \mathrm{~mol} / \mathrm{L}$ in $0.1 \mathrm{~mol} / \mathrm{L} \mathrm{NaOH})$ (Fluka Chemie GmbH, Buchs, Switzerland) was added and mixed. The contents were incubated at $90^{\circ} \mathrm{C}$ for $45 \mathrm{~min}$ in a water bath. Previously cooled (on ice to prevent further reaction) TBARS were extracted once with $1,000 \mu \mathrm{L}$ of $n$-butanol (Sigma-Aldrich Co.). The upper butanol phase was read at $535 \mathrm{~nm}$ and $572 \mathrm{~nm}$ to correct for baseline absorption using a Shimadzu UV-1601 spectrophotometer (Shimadzu Corp., Kyoto, Japan). Malondialdehyde equivalents (TBARS) quantification was done with the calibration curve.

\section{Plasma total antioxidant status}

Plasma total antioxidant status (TAS) levels were quantified using 2,2'-azino-bis (3-ethylbenzthiazoline-6-sulfonic acid) (ABTS) (Randox Laboratories Ltd), which is incubated with a peroxidase to produce the radical cation $\mathrm{ABTS}^{+}$. The bluish green staining of the $\mathrm{ABTS}^{+}$cation is relatively stable and measured at $600 \mathrm{~nm}$; antioxidants present in the plasma cause suppression of this color production to a degree that is proportional to the concentration. The kinetics reaction was measured with a Shimadzu UV-1601 spectrophotometer (Shimadzu Corp., Kyoto, Japan).

\section{Red blood cell superoxide dismutase}

In this method, superoxide radicals are generated by employing xanthine and xanthine oxidase. The formed radical reacts with 2-(4-iodophenyl)-3-(4-nitrophenol)-5phenyltetrazolium chloride to form a red formazan color, which is measured at $505 \mathrm{~nm}$. The superoxide dismutase (SOD) in the sample causes the inhibition of this reaction; the SOD activity is proportional to the degree of inhibition of the reaction (Randox Laboratories Ltd). Kinetics were measured with a Shimadzu UV-1601 spectrophotometer (Shimadzu Corp).

\section{Red blood cell glutathione peroxidase}

The glutathione peroxidase (GPx) catalyzes glutathione (GSH) oxidation by cumene hydroperoxide. In the presence of glutathione reductase (GR) and nicotinamide adenine dinucleotide phosphate (NADPH), oxidized GSH is immediately converted into the reduced form with a concomitant oxidation of NADPH to $\mathrm{NADP}^{+}$(Randox Laboratories Ltd). The decrease in absorbance is measured at $340 \mathrm{~nm}$; we used a Shimadzu UV-1601 spectrophotometer (Shimadzu Corp.).

We calculated the SOD/GPx ratio and the Antioxidant gap (AOGAP) using the following equation:

$$
\begin{aligned}
\text { AOGAP }= & (\text { TAS }-[(\text { albumin }(\mathrm{mmol}) \times 0.69) \\
& +\operatorname{uric} \operatorname{acid}(\mathrm{mmol})])^{24}
\end{aligned}
$$

\section{Oxidative stress score}

OxS was evaluated in relation to lipid peroxidation (LPO), SOD and GPx activities; TAS; SOD/GPx ratio; and AOGAP. On the basis of the 90th percentile of young healthy subjects, alternative cutoff values for each parameter were defined as follows: $\mathrm{LPO} \geq 0.340 \mathrm{mmol} / \mathrm{L} ; \mathrm{SOD} \leq 170 \mathrm{IU} / \mathrm{mL}$; $\mathrm{GPx} \leq 5,500 \mathrm{IU} / \mathrm{L} ; \mathrm{TAS} \leq 0.9 \mathrm{mmol} / \mathrm{L} ; \mathrm{SOD} / \mathrm{GPx} \geq 0.023$, and AOGAP $\leq 190 \mathrm{mmol} / \mathrm{L}$. An OxS-score was established ranging from 1 to 6 , representing the severity of biomarker modification; a score of 1 was given to each value beyond the cutoff. We categorized the subjects according to their OxS-score as follows: without $\mathrm{OxS}$ if $\mathrm{OxS}$-score was 0 to 2 and with $\mathrm{OxS}$ if OxS-score was 3 to $6 .{ }^{25}$

\section{Inflammatory cytokines}

Aliquots of serum sample were assayed by flow cytometry via the Cytometric Bead Array (CBA) Human Inflammatory 
Cytokine Kit (BD Biosciences, San Jose, CA, USA) to determine the levels of interleukin (IL)-6, IL-8, IL-10, and tumor necrosis factor-alpha (TNF- $\alpha){ }^{26}$

\section{TC exercise training program}

The training program followed the guidelines established by $\mathrm{Li}$ et $\mathrm{al}^{19}$ for the practice of "Eight-form easy Tai chi for elderly adults," which is 5 days a week, in sessions of 50 min each under the supervision of a qualified instructor for 6 months.

The participants had a 10-min warm-up, 30 min of practicing easy TC movements/postures, and a 5-min cooldown. Five-minute breaks between practice sessions were given to allow learners to rest and interact socially. After the warm-up, the participants held each single movement of the "Eight-form easy Tai chi for elderly adults" statically (in a stationary position) for a period of 5 to $10 \mathrm{~s}$.

The "Eight-form easy Tai chi for elderly adults" to high stance is as follows: (i) Commencing form: both hands rise to shoulder level; (ii) curving back arms (repulse monkey): right, left; 3 times each side; (iii) stepping sideways and moving arms (Grasp Peacock's Tail: ward off, rollback, press, push): to the left, then to the right; (iv) moving hands (wave hands like "clouds moving in the sky"): left side leads, 3 times; (v) Diagonal strides (fair lady works at shuttles): left, then right; (vi) Standing on one leg (golden cock stands on one leg): right, then left; (vii) stepping and pushing (brush knees and twist steps): left, then right; (viii) closing form: both hands fall to the side, left leg drawn to the right leg.

Each movement was practiced repetitively (8-10 repetitions) at a slow, self-controlled speed. After some successful initial practice of these static and moving positions, learners/ performers are ready to move on to linking the eight postures of easy TC in a sequential, continuous manner. Each daily session consisted of a minimum of five sets of easy TC, along with repeated practice of each movement.

\section{Statistical analysis}

Data analyses proceeded as follows: first, a descriptive analysis was performed to calculate the means and standard error (SE) of the outcomes over time. Second, a repeated measures multivariate analysis of variance was conducted to investigate the effects of the $\mathrm{TC}$ training program on changes of biochemical parameters, OxS and proinflammatory markers over time. The between-subject factor was each group (ie, the EG versus the CG), and the withinsubject factor was time (baseline versus 6 months). ${ }^{27}$ To compare the frequency of $\mathrm{OxS}$ pre- and post-intervention, we used a chi-squared test. In the present study, the significance level $(\alpha)$ was set at $<0.05$ for all statistical analyses. We used the statistical analysis program IBM SPSS Statistics 20.0.

\section{Results}

Over the course of 6 months, no statistically significant differences were observed (Table 1) in the TC practice group compared with the CG in the following measurements: RHR, DBP, SBP, MAP, RHR-SBP product, RHR-MAP product, body weight, and BMI.

Regarding the biochemical parameters, a statistically significant decrease in $\mathrm{HbA} 1 \mathrm{c}$ concentration was observed in the TC group compared with the CG $(p<0.05)$, whereas no statistically significant differences were found in the blood levels of triglycerides, cholesterol, HDL, LDL, and glucose between the TC and CGs (Table 2).

In respect to the OxS markers, the group that practiced TC showed a statistically significant increase in total antioxidant status and a decrease in the OxS grade compared with

Table I Clinical and anthropometric parameters by study group

\begin{tabular}{|c|c|c|c|}
\hline Parameters & $\begin{array}{l}\text { Control } \\
(n=37)\end{array}$ & $\begin{array}{l}\text { Tai chi } \\
(n=48)\end{array}$ & p-value \\
\hline Age (years) & $68.2 \pm 6.6$ & $67.4 \pm 4.7$ & 0.77 \\
\hline \multicolumn{4}{|l|}{ Weight (kg) } \\
\hline Baseline & $68.0 \pm 1.5$ & $69.7 \pm 1.7$ & \\
\hline Six months & $68.9 \pm 1.6$ & $69.3 \pm 1.8$ & 0.49 \\
\hline \multicolumn{4}{|l|}{ BMI } \\
\hline Baseline & $28.3 \pm 0.5$ & $30.2 \pm 0.7$ & \\
\hline Six months & $28.7 \pm 0.6$ & $30.0 \pm 0.7$ & 0.83 \\
\hline \multicolumn{4}{|c|}{ RHR (beats per minute) } \\
\hline Baseline & $64.0 \pm 8.3$ & $64.3 \pm 10.9$ & \\
\hline Six months & $67.6 \pm 10.1$ & $67.4 \pm 11.9$ & 0.84 \\
\hline \multicolumn{4}{|l|}{ SBP (mmHg) } \\
\hline Baseline & $131.8 \pm 2.6$ & $127.6 \pm 3.0$ & \\
\hline Six months & $132.8 \pm 2.1$ & $129.4 \pm 2.4$ & 0.57 \\
\hline \multicolumn{4}{|l|}{ DBP (mmHg) } \\
\hline Baseline & $76.3 \pm 1.0$ & $78.6 \pm 1.9$ & \\
\hline Six months & $80.8 \pm 1.3$ & $79.5 \pm 1.4$ & 0.53 \\
\hline \multicolumn{4}{|l|}{ MAP (mmHg) } \\
\hline Baseline & $6,026.7 \pm 1,019.5$ & $6,147.5 \pm 1,377.7$ & \\
\hline Six months & $6,525.5 \pm 1,001.9$ & $6,538.3 \pm 1,452.4$ & 0.79 \\
\hline \multicolumn{4}{|c|}{ RHR-SBP product } \\
\hline Baseline & $8,389.1 \pm 1,540.0$ & $8,301.1 \pm 2,029.0$ & \\
\hline Six months & $8,84 I . I \pm I, 487.3$ & $8,8 \mid 4.1 \pm 2,024.0$ & 0.88 \\
\hline \multicolumn{4}{|c|}{ RHR-MAP product } \\
\hline Baseline & $6,026.7 \pm 1,019.5$ & $6,147.5 \pm 1,377.7$ & \\
\hline Six months & $6,525.5 \pm 1,001.9$ & $6,538.3 \pm 1,452.4$ & 0.79 \\
\hline
\end{tabular}

Notes: Values are means \pm SE. Repeated measures analysis of variance.

Abbreviations: DBP, diastolic blood pressure; MAP, mean arterial pressure; RHR, resting heart rate; RHR-MAP product, resting heart rate $\times$ mean arterial pressure; RHR-SBP product, resting heart rate $\times$ systolic blood pressure; $S E$, standard error; SBP, systolic blood pressure. 
Table 2 Biochemical parameters by study group

\begin{tabular}{|c|c|c|c|}
\hline Parameters & $\begin{array}{l}\text { Control } \\
(n=37)\end{array}$ & $\begin{array}{l}\text { Tai chi } \\
(n=48)\end{array}$ & $p$-value \\
\hline \multicolumn{4}{|c|}{ Glucose (mg/dL) } \\
\hline Baseline & $113.3 \pm 4.2$ & $121.8 \pm 3.4$ & \\
\hline $\begin{array}{c}\text { Six months } \\
\text { Urate (mg/dL) }\end{array}$ & $109.4 \pm 4.3$ & $120.0 \pm 3.5$ & 0.73 \\
\hline Baseline & $4.4 \pm 0.2$ & $5.2 \pm 0.3$ & \\
\hline $\begin{array}{c}\text { Six months } \\
\text { Albumin }(\mathrm{mg} / \mathrm{d}\end{array}$ & $4.8 \pm 0.4$ & $5.4 \pm 0.2$ & 0.67 \\
\hline Baseline & $4.5 \pm 0.1$ & $4.4 \pm 0.09$ & \\
\hline $\begin{array}{l}\text { Six months } \\
\text { Cholesterol }(\mathrm{m}\end{array}$ & $4.8 \pm 0.07$ & $4.7 \pm 0.04$ & 0.87 \\
\hline Baseline & $202.2 \pm 6.7$ & $209.4 \pm 4.9$ & \\
\hline $\begin{array}{c}\text { Six months } \\
\text { HDL-C (mg/dL }\end{array}$ & $192.2 \pm 7.3$ & $210.1 \pm 4.6$ & 0.46 \\
\hline Baseline & $45.5 \pm 1.4$ & $42.6 \pm 0.9$ & \\
\hline $\begin{array}{l}\text { Six months } \\
\text { LDL-C (mg/dL }\end{array}$ & $53.9 \pm 1.7$ & $50.8 \pm 1.4$ & 0.34 \\
\hline Baseline & $120.0 \pm 5.4$ & $132.6 \pm 3.2$ & \\
\hline $\begin{array}{l}\text { Six months } \\
\text { HbAlc (\%) }\end{array}$ & $100.2 \pm 5.7$ & $120.1 \pm 3.6$ & 0.13 \\
\hline Baseline & $7.0 \pm 0.3$ & $8.2 \pm 0.3$ & \\
\hline Six months & $6.9 \pm 0.4$ & $7.3 \pm 0.3$ & 0.03 \\
\hline
\end{tabular}

Notes: Values are means \pm SE. Repeated measures analysis of variance. Abbreviations: HbAIc, glycosylated hemoglobin; HDL-C, high-density lipoprotein cholesterol; LDL-C, low-density lipoprotein cholesterol; SE, standard error.

the CG $(p<0.05)$ (Table 3). In this sense, the percentage of subjects with $\mathrm{OxS}$ detected in the TC group showed a statistically significant decrease (baseline, $56 \%$ vs TC, $23 \%$, $p<0.01$ ) in comparison with the CG (Table 4).

Table 3 Oxidative stress markers by study group

\begin{tabular}{|c|c|c|c|}
\hline Markers & $\begin{array}{l}\text { Control } \\
(n=37)\end{array}$ & $\begin{array}{l}\text { Tai chi } \\
(n=48)\end{array}$ & $p$-value \\
\hline \multicolumn{4}{|c|}{ Lipoperoxides ( $\mu \mathrm{mol} / \mathrm{L})$} \\
\hline Baseline & $0.29 \pm 0.02$ & $0.30 \pm 0.01$ & \\
\hline Six months & $0.34 \pm 0.01$ & $0.33 \pm 0.01$ & 0.82 \\
\hline \multicolumn{4}{|l|}{ SOD (Ul/mL) } \\
\hline Baseline & $179.0 \pm 1.4$ & $178.7 \pm 1.8$ & \\
\hline $\begin{array}{l}\text { Six months } \\
\text { GPx (UI/L) }\end{array}$ & $173.7 \pm 2.1$ & $173.9 \pm 1.2$ & 0.54 \\
\hline Baseline & $7,670 \pm 856$ & $7,427 \pm 509$ & \\
\hline $\begin{array}{l}\text { Six months } \\
\text { SOD/GPx }\end{array}$ & $9,564 \pm 794$ & $9,616 \pm 501$ & 0.96 \\
\hline Baseline & $0.030 \pm 0.005$ & $0.028 \pm 0.002$ & \\
\hline $\begin{array}{c}\text { Six months } \\
\text { TAS (mmol/L) }\end{array}$ & $0.025 \pm 0.004$ & $0.021 \pm 0.00 I$ & 0.61 \\
\hline Baseline & $0.85 \pm 0.02$ & $1.03 \pm 0.04$ & \\
\hline Six months & $0.98 \pm 0.04$ & $1.28 \pm 0.02$ & $<0.01$ \\
\hline \multicolumn{4}{|c|}{ AOGAP $(\mu \mathrm{mol} / \mathrm{L})$} \\
\hline Baseline & $171.7 \pm 23.6$ & $228.3 \pm 38.0$ & \\
\hline Six months & $356.1 \pm 60.0$ & $427.5 \pm 32.3$ & 0.56 \\
\hline \multicolumn{4}{|l|}{ OxS-score } \\
\hline Baseline & $2.69 \pm 0.2$ & $2.28 \pm 0.2$ & \\
\hline Six months & $2.23 \pm 0.2$ & $1.11 \pm 0.1$ & $<0.01$ \\
\hline
\end{tabular}

Notes: Values are means \pm SE. Repeated measures analysis of variance.

Abbreviations: AOGAP, antioxidant GAP; GPx, glutathione peroxidase; OxSscore, oxidative stress score; SE, standard error; SOD, superoxide dismutase; TAS, total antioxidant status.
Table 4 Frequency of oxidative stress pre- and post-intervention

\begin{tabular}{|c|c|c|c|c|}
\hline & \multicolumn{2}{|l|}{ Control } & \multicolumn{2}{|l|}{ Tai chi } \\
\hline & $\begin{array}{l}\text { Baseline, } \\
\text { n (\%) }\end{array}$ & $\begin{array}{l}\text { Six months, } \\
\text { n (\%) }\end{array}$ & $\begin{array}{l}\text { Baseline, } \\
\text { n (\%) }\end{array}$ & $\begin{array}{l}\text { Six months, } \\
\text { n (\%) }\end{array}$ \\
\hline Without OxS & $14(38)$ & 21 (57) & 2I (44) & 37 (77) \\
\hline With OxS & $23(62)$ & $16(43)$ & $27(56)$ & II (23)* \\
\hline
\end{tabular}

Note: Chi-squared test: ${ }^{*} p<0.01$.

Abbreviation: OxS, oxidative stress.

In the measurement of markers of chronic inflammation, a statistically significant decrease in IL-8 was found in the group that practiced TC (baseline: $3.6 \pm 0.3$ vs TC: $1.6 \pm 0.2 \mathrm{pg} / \mathrm{mL}, p<0.01$ ) (Table 5).

\section{Discussion}

Some chronic diseases of high prevalence in the elderly, such as diabetes mellitus type 2, cardiovascular diseases, cancer and Alzheimer's disease have been linked with MetS, in addition to accelerated aging and fragility. Likewise, OxS and chronic inflammation are biochemical alterations related to the etiology and complications of MetS and the abovementioned diseases..$^{28,29}$

It has been proposed that moderate physical exercise has a positive impact on $\mathrm{OxS}$ and inflammation during aging. ${ }^{30,31}$ In this sense, it has been proven that regular practice of moderate physical exercise has a positive effect on the aging process, thus contributing to minimized biochemical changes related to aging and decreasing the risk of chronic diseases, therefore promoting health in aging individuals and increasing longevity. ${ }^{32,33}$

Currently, the practice of TC is common to prevent and control chronic diseases of high prevalence in old age. ${ }^{34}$

Table 5 Inflammatory markers by study group

\begin{tabular}{|c|c|c|c|}
\hline Markers & $\begin{array}{l}\text { Control } \\
(n=37)\end{array}$ & $\begin{array}{l}\text { Tai chi } \\
(n=48)\end{array}$ & $p$-value \\
\hline \multicolumn{4}{|l|}{ IL-6 (pg/mL) } \\
\hline Pre-intervention & $1.24 \pm 0.1$ & $0.97 \pm 0.1$ & \\
\hline Post-intervention & $1.39 \pm 0.2$ & $1.28 \pm 0.2$ & 0.63 \\
\hline \multicolumn{4}{|l|}{ IL-8 (pg/mL) } \\
\hline Pre-intervention & $4.6 \pm 0.5$ & $3.6 \pm 0.3$ & \\
\hline Post-intervention & $5.9 \pm 0.7$ & $1.6 \pm 0.2$ & 0.01 \\
\hline \multicolumn{4}{|l|}{ IL-I0 (pg/mL) } \\
\hline Pre-intervention & $1.5 \pm 0.05$ & $1.6 \pm 0.02$ & \\
\hline Post-intervention & $1.4 \pm 0.08$ & $1.0 \pm 0.10$ & 0.17 \\
\hline \multicolumn{4}{|l|}{ TNF- $\alpha(p g / m L)$} \\
\hline Pre-intervention & $1.9 \pm 0.02$ & $2.1 \pm 0.2$ & \\
\hline Post-intervention & $1.8 \pm 0.05$ & $2.0 \pm 0.2$ & 0.87 \\
\hline \multicolumn{4}{|l|}{ CRP (mg/dL) } \\
\hline Pre-intervention & $0.21 \pm 0.05$ & $0.36 \pm 0.04$ & \\
\hline Post-intervention & $0.23 \pm 0.03$ & $0.39 \pm 0.04$ & 0.51 \\
\hline
\end{tabular}

Notes: Values are means \pm SE. Repeated measures analysis of variance.

Abbreviations: CRP, C-reactive protein; IL-10, interleukin I0; IL-6, interleukin 6; IL-8, interleukin 8; SE, standard error; TNF- $\alpha$, tumor necrosis factor alpha. 
In this sense, in some studies, a positive effect of TC training on RHR, SBP, DBP, MAP, and RHR-SBP product has been reported; ${ }^{35-37}$ however, in our study, no significant changes were observed in these parameters. This may be due to training time. In this regard, there is a study that has reported a positive effect on cardiovascular parameters after 2 years of TC training. ${ }^{38}$

On the other hand, in the present study, a statistically significant decrease in $\mathrm{HbA} 1 \mathrm{c}$ concentration was observed in the CT group $(p<0.05)$. In this respect, this finding is congruent with what has been reported in some studies, suggesting that the hypoglycemic effect of CT is due to this type of physical activity, incorporating breathing techniques and body movement that burn calories. ${ }^{12,39}$ However, the effectiveness of the practice of TC for the control of type 2 diabetes mellitus is still controversial, so further large-scale studies are needed to investigate the long-term efficacy of TC. ${ }^{40}$

Regarding control of dyslipidemias after TC training, a decrease in blood levels of triglycerides, total cholesterol, and LDL-C has been observed, as well as increased concentration of HDL-C. ${ }^{15,17}$

However, in the present study, no statistically significant differences were observed in the biochemical markers mentioned above. In this sense, Pan et $\mathrm{al}^{41}$ reported in a meta-analysis of randomized controlled trials a statistically significant decrease in blood triglyceride concentration but found no statistically significant differences in total cholesterol, LDL-C, and HDL-C concentrations. Other studies support the proposal that there are no changes in parameters related to metabolic syndrome such as dyslipidemias, hyperglycemia, and waist circumference after TC training. ${ }^{35,37}$ These inconsistencies justify carrying out longitudinal long-term studies on the effect of TC on the biochemical parameters related to metabolic syndrome in older adults.

Some studies have shown that MetS is associated with increased OxS. ${ }^{1,28}$ In this sense, our research group, in a previous study, found that the number of MetS components is a significant risk factor in the development of OxS. ${ }^{1}$ Likewise, it has been demonstrated that the practice of moderate physical exercise has an antioxidant and anti-inflammatory effect in adult patients with MetS.9,42 In addition, an antioxidant effect of TC practice has been observed in healthy adults and older adults. ${ }^{15-18}$ However, there are no studies on the antioxidant effect of TC in older adults with MetS. In the present study, we found a statistically significant increase in total antioxidant status activity $(p<0.01)$. This finding is similar to that reported in a study of healthy middle-aged people (40-45 years of age), although in this study, they also observed a statistically significant decrease in LPO concentration and a statistically significant increase in SOD activity, ${ }^{43}$ in contrast to our results, where we found no statistically significant differences in LPO concentration and SOD activity between the group that performed TC and the control. These differences may be because our study was carried out in older persons with alterations of MetS. However, it is important to note that $\mathrm{OxS}$ is a dynamic process characterized by a biochemical imbalance caused by excessive production of reactive species, mainly of oxygen, that cannot be counteracted by antioxidant systems which causes oxidation of biomolecules. Therefore, OxS cannot be measured by isolated biochemical markers; as a result, an OxS score to measure the severity of OxS has been developed. ${ }^{1,25}$ In this sense, our findings suggest that the antioxidant effect of $\mathrm{TC}$ is maintained in the elderly with MetS, with a $50 \%$ decrease in $\mathrm{OxS}$ score.

The mechanism suggests that the antioxidant effect of physical exercise changes the body's redox balance in favor of more alkaline conditions in the cell. The reactive species that generate during physical exercise act as a signal that is necessary for the activation of the MAPK proteins (p38 and ERK1/ERK2), which in turn activate the transcription factor sensitive to the redox state, nuclear factor kappa $B$ $(\mathrm{NF}-\kappa \beta)$, via activation of the kinase that phosphorylates the inhibitor of this factor (I $\kappa)$ ). Once freed from its inhibitor, $\mathrm{NF}-\kappa \beta$ migrates toward the nucleus where it can promote the synthesis of various antioxidant enzymes, such as MnSOD, iNOS, and glutamylcysteine synthetase. ${ }^{44}$ In this sense, the positive effects of TC may be due to the combination of various mechanisms, including the previously mentioned signaling. In addition to being a moderate form of exercise, it provides a relaxing psychological effect, similar to that reported in transcendental meditation. The practice of deep meditation (which is an essential characteristic of TC) is associated with lower levels of LPO, which has been explained as an effect of lower activity in the sympathetic nervous system. Another hypothesis suggests that profound meditation increases the levels of the hormone dehydroepiandrosterone, a marker of successful aging that has been linked with an increase in the activity of antioxidant enzymes. ${ }^{45,46}$

The practice of moderate physical exercise, including TC, has a positive effect on markers of chronic inflammation. ${ }^{9,11}$ In this regard, our study showed a statistically significant decrease in IL-8 levels in the TC group compared with the CG. In this sense, the IL-8 is a proinflammatory chemokine associated with the promotion of neutrophil chemotaxis and degranulation. The induction of IL-8 signaling activates multiple upstream signaling pathways that (i) impinge on gene expression via regulation of numerous transcription 
factor activities, (ii) modulate the cellular proteome at the level of translation, and/or (iii) affect the organization of the cell cytoskeleton through posttranslational regulation of regulatory proteins. ${ }^{47}$ In this regard, it has been demonstrated that increased serum concentrations of IL-8 are associated with poor memory and speed domain performance as well as decreased in motor function in elderly subjects. ${ }^{48}$ For this reason, the practice of TC also has an effect on maintaining and improving memory during the aging process.

Although the molecular changes related to the practice of TC linked to health are not completely clear, Buric et al (2017), ${ }^{49}$ as a result of a systematic review, conclude that TC as well as other mind-body interventions (MBIs) are associated with the downregulation of NF- $\kappa \beta$ pathway. This is the opposite of the effects of chronic stress on gene expression and suggests that MBI practices may lead to a reduced risk of inflammation-related diseases. In this sense, MBIs have been shown to have a positive effect on the control of MetS; 50 even the practice of TC for the prevention of MetS has been recommended. ${ }^{11}$

In conclusion, our findings suggest that the practice of TC in the elderly with MetS has antioxidative and hypoglycemic effects, so it could be recommended as a coadjuvant therapy for these patients. Nevertheless, it is important to note the limitations of the research, including the lack of monitoring of the type of alimentation, daily activities and psychosocial conditions faced by the participants during the study, all of which could influence the results.

\section{Acknowledgments}

This work was supported by Dirección General de Asuntos del Personal Académico, Universidad Nacional Autónoma de México (DGAPA, UNAM), PAPIIT IN306213-2 \& Posgrado en Ciencias Biológicas, UNAM. We appreciate the support of Itzen Aguiñiga-Sánchez for measured of inflammatory cytokines.

\section{Disclosure}

The authors report no conflicts of interest in this work.

\section{References}

1. Sánchez-Rodríguez MA, Martínez-Cruz M, Correa-Muñoz E, Mendoza-Núñez VM. Relationship between metabolic syndrome components and oxidative stress in elderly community-dwelling Mexicans. Ann Nutr Metab. 2010;56(4):302-307.

2. Grundy SM. Metabolic syndrome pandemic. Arterioscler Thromb Vasc Biol. 2008;28(4):629-636.

3. Lechleitner M. Obesity and the metabolic syndrome in the elderly - a mini-review. Gerontology. 2008;54(5):253-259.

4. Levin BE, Llabre MM, Dong C, et al. Modeling metabolic syndrome and its association with cognition: the Northern Manhattan study. J Int Neuropsychol Soc. 2014;20(10):951-960.
5. Lin F, Roiland R, Chen DG, Qiu C. Linking cognition and frailty in middle and old age: metabolic syndrome matters. Int J Geriatr Psychiatry. 2015;30(1):64-71.

6. Cufí S, Vazquez-Martin A, Oliveras-Ferraros C, Martin-Castillo B, Joven J, Menendez JA. Metformin against TGF $\beta$-induced epithelialto-mesenchymal transition (EMT): from cancer stem cells to agingassociated fibrosis. Cell Cycle. 2010;9(22):4461-4468.

7. Cardinali DP, Hardeland R. Inflammaging, metabolic syndrome and melatonin: a call for treatment studies. Neuroendocrinology. 2017; 104(4):382-397.

8. Szarc vel Szic K, Declerck K, Vidaković M, Vanden Berghe W. From inflammaging to healthy aging by dietary lifestyle choices: is epigenetics the key to personalized nutrition? Clin Epigenetics. 2015;7:33.

9. Farinha JB, Steckling FM, Stefanello ST, et al. Response of oxidative stress and inflammatory biomarkers to a 12 -week aerobic exercise training in women with metabolic syndrome. Sports Med Open. 2015; 1(1): 19 .

10. Hui SS, Xie YJ, Woo J, Chi-Yui Kwok T. Effects of Tai Chi and walking exercises on weight loss, metabolic syndrome parameters, and bone mineral density: a cluster randomized controlled trial. Evid Based Complement Alternat Med. 2015;2015:976123.

11. Chen M, He M, Min X, et al. Different physical activity subtypes and risk of metabolic syndrome in middle-aged and older Chinese people. PLoS One. 2013;8(1):e53258.

12. Liu X, Miller YD, Burton NW, Brown WJ. A preliminary study of the effects of Tai Chi and Qigong medical exercise on indicators of metabolic syndrome, glycaemic control, health-related quality of life, and psychological health in adults with elevated blood glucose. Br J Sports Med. 2010;44(10):704-709.

13. Li JX, Hong Y, Chan KM. Tai chi: physiological characteristics and beneficial effects on health. Br J Sports Med. 2001;35(3):148-156.

14. Hong Y. Tai Chi Chuan. State of the art in international research. Medicine Sports Science. Hong Kong: Basel, Karger; 2008.

15. Rosado-Pérez J, Santiago-Osorio E, Ortiz R, Mendoza-Núñez VM. Tai chi diminishes oxidative stress in Mexican older adults. $J$ Nutr Health Aging. 2012;16(7):642-646.

16. Mendoza-Núñez VM, Hernández-Monjaraz B, Santiago-Osorio E, Betancourt-Rule JM, Ruiz-Ramos M. Tai Chi exercise increases SOD activity and total antioxidant status in saliva and is linked to an improvement of periodontal disease in the elderly. Oxid Med Cell Longev. 2014; 2014:603853.

17. Chen SC, Ueng KC, Lee SH, Sun KT, Lee MC. Effect of T'ai Chi exercise on biochemical profiles and oxidative stress indicators in obese patients with type 2 diabetes. J Altern Complement Med. 2010; 16(11):1153-1159.

18. Huang XY, Eungpinichpong W, Silsirivanit A, Nakmareong S, Wu XH. Tai chi improves oxidative stress response and DNA damage/repair in young sedentary females. J Phys Ther Sci. 2014;26(6):825-829.

19. Li F, Fisher KJ, Harmer P, Shirai M. A simpler eight-form easy Tai chi for elderly adults. J Aging Phys Act. 2003;11:206-218.

20. National Cholesterol Education Program (NCEP) Expert Panel on Detection, Evaluation, and Treatment of High Blood Cholesterol in Adults (Adult Treatment Panel III): Third Report of the National Cholesterol Education Program (NCEP) Expert Panel on Detection, Evaluation, and Treatment of High Blood Cholesterol in Adults (Adult Treatment Panel III) final report. Circulation. 2002;106:3143-3421.

21. Secretaría de Salud. Norma Oficial Mexicana NOM-030-SSA-1999, para la prevención, tratamiento y control de la hipertensión arterial. Mexico City: Secretaría de Salud, 1999. [Ministry of Health. Official Mexican Standard NOM-030-SSA-1999, for the prevention, treatment and control of arterial hypertension. Mexico City: Ministry of Health, 1999]. Spanish.

22. Friedewald WT, Levy RI, Fredrickson DS. Estimation of the concentration of low density lipoprotein cholesterol in plasma, without use of the preparative ultracentrifuge. Clin Chem. 1972;18:499-502.

23. Jentzsch AM, Bachmann H, Fürst P, Biesalski HK. Improved analysis of malondialdehyde in human body fluids. Free Radic Biol Med. 1996; 20:251-256. 
24. Miller NJ. Nonvitamin plasma antioxidants. In: Armstrong D, editor. Free Radical and Antioxidant Protocols. Totowa, NJ: Humana Press; 1998:285-297. Available from: https://doi.org/10.1385/0-89603-4720:285. Accessed February 28, 2018.

25. Sánchez-Rodríguez MA, Ruiz-Ramos M, Mendoza-Núñez VM. Proposal of a construct to measure severity of oxidative stress. Free Radic Biol Med. 2006;41:S29.

26. Cook EB, Stahl JL, Lowe L, et al. Simultaneous measurement of six cytokines in a single sample of human tears using microparticle-based flow cytometry: allergics vs. non-allergics. J Immunol Methods. 2001; 254(1-2):109-118.

27. Park E, Cho M, Ki CS. Correct use of repeated measures analysis of variance. Korean J Lab Med. 2009;29(1):1-9.

28. Bonomini F, Rodella LF, Rezzani R. Metabolic syndrome, aging and involvement of oxidative stress. Aging Dis. 2015;6(2):109-120.

29. Yaffe K. Metabolic syndrome and cognitive disorders: is the sum greater than its parts? Alzheimer Dis Assoc Disord. 2007;21(2):167-171.

30. McArdle A, Jackson MJ. Exercise, oxidative stress and ageing. J Anat. 2000;197:539-541.

31. Radak Z, Chung HY, Goto S. Exercise and hormesis: oxidative stressrelated adaptation for successful aging. Biogerontology. 2005;6:71-75.

32. Bassey EJ. The benefits of exercise for the health of older people. Rev Clin Gerontol. 2000;10:17-31

33. Singh MAF. Exercise and aging. Clin Geriatr Med. 2004;20:201-221.

34. Huston P, McFarlane B. Health benefits of tai chi: what is the evidence? Can Fam Physician. 2016;62(11):881-890.

35. Yu R, Woo J, Chan AS, Sze SL. A Chinese Chan-based mind-body intervention improves psychological well-being and physical health of community-dwelling elderly: a pilot study. Clin Interv Aging. 2014;9: 727-736.

36. Yeh GY, Wang C, Wayne PM, Phillips RS. The effect of tai chi exercise on blood pressure: a systematic review. Prev Cardiol. 2008;11(2):82-89.

37. Sun J, Buys N. Community-based mind-body meditative Tai Chi program and its effects on improvement of blood pressure, weight, renal function, serum lipoprotein, and quality of life in Chinese adults with hypertension. Am J Cardiol. 2015;116(7):1076-1081.

38. Figueroa MA, DeMeersman RE, Manning J. The autonomic and rate pressure product responses of Tai chi practitioners. $N$ Am J Med Sci. 2012;4:270-275.
39. Pai LW, Li TC, Hwu YJ, Chang SC, Chen LL, Chang PY. The effectiveness of regular leisure-time physical activities on long-term glycemic control in people with type 2 diabetes: A systematic review and metaanalysis. Diabetes Res Clin Pract. 2016;113:77-85.

40. Yan JH, Gu WJ, Pan L. Lack of evidence on Tai Chi-related effects in patients with type 2 diabetes mellitus: a meta-analysis. Exp Clin Endocrinol Diabetes. 2013;121(5):266-271.

41. Pan XH, Mahemuti A, Zhang XH, et al. Effect of Tai Chi exercise on blood lipid profiles: a meta-analysis of randomized controlled trials. J Zhejiang Univ Sci B. 2016;17(8):640-648.

42. Stensvold D, Slørdahl SA, Wisløff U. Effect of exercise training on inflammation status among people with metabolic syndrome. Metab Syndr Relat Disord. 2012;10(4):267-272.

43. Niu A. Effect of "Tai Chi" exercise on antioxidant enzymes activities and immunity function in middle-aged participants. Afr $J$ Tradit Complement Altern Med. 2016;13(5):87-90.

44. Gomez-Cabrera MC, Domenech E, Viña J. Moderate exercise is an antioxidant: upregulation of antioxidant genes by training. Free Radic Biol Med. 2008;44(2):126-131.

45. Epel E, Daubenmier J, Moskowitz JT, Folkman S, Blackburn E. Can meditation slow rate of cellular aging? Cognitive stress, mindfulness, and telomeres. Ann N Y Acad Sci. 2009;1172:34-53.

46. Mahagita $C$. Roles of meditation on alleviation of oxidative stress and improvement of antioxidant system. JMed Assoc Thai. 2010;93(Suppl 6): S242-S254

47. Waugh DJ, Wilson C. The interleukin-8 pathway in cancer. Clin Cancer Res. 2008;14(21):6735-6741.

48. Baune BT, Ponath G, Golledge J, et al. Association between IL-8 cytokine and cognitive performance in an elderly general population the MEMO-Study. Neurobiol Aging. 2008;29(6):937-944.

49. Buric I, Farias M, Jong J, Mee C, Brazil IA. What is the molecular signature of mind-body interventions? A systematic review of gene expression changes induced by meditation and related practices. Front Immunol. 2017;8:670.

50. Anderson JG, Taylor AG. The metabolic syndrome and mind-body therapies: a systematic review. J Nutr Metab. 2011;2011:276419.
Clinical Interventions in Aging

\section{Publish your work in this journal}

Clinical Interventions in Aging is an international, peer-reviewed journal focusing on evidence-based reports on the value or lack thereof of treatments intended to prevent or delay the onset of maladaptive correlates of aging in human beings. This journal is indexed on PubMed Central, MedLine,

\section{Dovepress}

CAS, Scopus and the Elsevier Bibliographic databases. The manuscript management system is completely online and includes a very quick and fair peer-review system, which is all easy to use. Visit http://www.dovepress. com/testimonials.php to read real quotes from published authors. 